»eine Professionalisierung der Männer des Krieges [...], die Bildung eines Waffenarsenals; zweitens eine ständige bewaffnete Struktur, die in der Lage ist, als Rahmen für besondere Rekrutierungen in Kriegszeiten zu dienen; drittens eine Ausstattung an Festungen und Transportmitteln; viertens schließlich ein Wissen, eine taktische Reflexion, Typen von Manövern, Schemata von Angriff und Verteidigung, kurz eine ganze eigentümliche und eigenständige Reflexion auf militärische Angelegenheiten und mögliche Kriege.«(Foucault [1978] 2006: 441)

Die Analyse eines Dispositivs ähnelt somit einer Analyse der Taktiken und Strategien, die die widerstehende Kriegspartei überblicken muss, um sich erfolgreich verteidigen, den Krieg sabotieren oder mit List und Tücke gewinnen zu können.

\title{
2 Wissenschaftliche Rezeption Foucaults Dispositivbegriffs
}

Die Dispositivanalyse wurde im Vergleich zu anderen analytischen Instrumenten Foucaults deutlich beschränkter rezipiert. Dem liegt einerseits die Tatsache zugrunde, dass Foucault seinen Begriff keineswegs als ein methodisches Werkzeug beschrieben hatte, sondern mit ihm einen geschichtlichen Blick auf konkrete Regierungsverhältnisse ermöglichen wollte; sei es auf die Entstehung der Staatsräson mit den Dispositiven Diplomatie-Militär und Polizei, sei es auf die Entwicklung des Sexualitätsdispositivs oder des Dispositivs der Delinquenz, die als Machteffekte die Schattenseiten und Wirkungsmechanismen von problematisierten gesellschaftlichen Feldern - der Sicherheit, der Sexualität, der Kriminalität - widerspiegelt haben.

Andererseits sorgte eine nie endgültige und nie klar zu dechiffrierende Zahl an Dispositiv-Elementen - Foucault favorisierte den Termin Ensembles - für eine Art wissenschaftliche Aversion gegenüber dem Begriff des Dispositivs, seiner Relevanz und seiner möglichen Anwendung. Denn der analytische Ausgang konnte und kann nie vorhergesehen werden, da das Dispositiv ein zeitlich und räumlich nicht abzugrenzendes Netz zwischen seinen Elementen herstellt und eine fortdauernde Entwicklung mit der »Prävalenz einer strategischen Zielsetzung« bildet (Foucault [1977] 1978c: 121; vgl. auch Gille 2013: 75). Mit der Dispositivanalyse eröffnet sich also ein risikoreiches Unternehmen mit nicht vorher definierbaren analytischen Vorkehrungen, und deswegen kann eher von einem Forschungsstil, als von einer Forschungsmethode gesprochen werden (vgl. Bührmann \& Schneider 2012). Nichtdestotrotz erfährt in den letzten Jahren seine Anwendung ein wachsendes Interesse. ${ }^{5}$ Um den Forschungsstil der Dispositivanalyse illustrieren und auf die eigene 
Dispositiv-Bestimmung hinausgehen zu können, werden nun einige Aspekte des Dispositivs, wie sie in der Dispositivforschung herausgearbeitet werden, reflektiert.

\subsection{Wirkungsweise der Dispositive}

Der Dispositiv-Begriff wird insbesondere in der interdisziplinären Diskursforschung aufgenommen. Dort wird zuerst seine Mehrdeutigkeit thematisiert. So ist das Dispositiv zuerst »ein Sachverhalt, der für spezifische Zwecke eingesetzt werden kann, der zur Verfügung steht. Ein Dispositiv ist hier allgemein ein Instrument, ein Werkzeug oder ein Apparat.« (Diaz-Bone \& Hartz 2017: 5) Gleichzeitig sind Dispositive im Plural »formierende, beeinflussende, gestaltende und so konstruierende Formationen, die daher ihre Wirkmächtigkeit in der materiellen und immateriellen Mitkonstruktion der Sachverhalte haben, auf die sie >zugreifen «." (a.a.O.: 6 [Herv. i. O.]) Dieser auf den ersten Blick konfusen Definition unterliegt die semantische Abstammung des Begriffs selbst. Denn seine Bedeutung ist »im Französischen noch reichhaltiger, da das französische Wort >dispositif ‘ auch die >Maßnahmeく, das `System oder die >Mittek bezeichnen kann. Alle diese weiteren Bezeichnungen haben gemeinsam, dass sie auch auf eine der Formierung und Anwendung unterliegende Absicht, Intentionalität, Planung oder Strategie verweisen.« (a.a.O.: 5 [Herv. i. O.]) Daher wird die Verbindung Sachverhalt-Formation in dem Begriff des Dispositivs möglich. ${ }^{6}$

Des Weiteren werden die Dispositive analytisch als Erweiterung der Diskurse betrachtet, weshalb es erforderlich ist, »ihre Erklärungsleistung im Rahmen einer nicht allein dispositivanalytischen Erklärung zu entwickeln, sondern regelmäßig in einer diskursanalytischen sund (!) dispositivanalytischen Untersuchungsanlage (Jäger \& Jäger 2007; Jäger 2015). «(a.a.O.: 9 [Herv. i. O.]) Dispositive stellen indessen die Möglichkeit dar, die Objekte gleichwie die »Steigerungen von Diskurseffekten wie die diskursive Anrufung von Subjekten und die Subjektivierungen - oder die Wechselwirkungen von diskursiven Praktiken und Dispositiven « (a.a.O.: 7) in die Analyse miteinzubeziehen. Dispositive sind also "nie als alleinige und selbstständige Analysen denkbar« (a.a.O.: 8), sondern sind als abgeleitete Realitäten bzw. Realitäten zweiter Art »einmal selbst Formationen, also mit aus vorgängigen Elementen organisierte, neue Realitäten, dann sind sie mit Absicht, Intention und unter strategischen Gesichtspunkten formiert worden und zuletzt sind sie wandelbar und

Methode eine wachsende Resonanz auch in den Kultur- und Sozialwissenschaften (vgl. Carstensen 2019), in der Literaturwissenschaft (vgl. Vomhof 2017) oder in der Kunstgeschichte (vgl. Frohne/Haberer/Urban 2019).

6 Für die alltagssprachlichen Konnotationen der französischen Begriffe dispositif und disposition siehe Link 2007: 219f. 
für andere Zwecke nutzbar, sumnutzbar und anders verwendbar (und eventuell aus Sicht ihrer ursprünglichen Formierenden gar >missbrauchbar $`$.« (ebd. [Herv. i. O.])

Was daraus folgt, ist, dass ihre Untersuchung nur dann erfolgreich sein kann, »wenn man die Prozesse ihrer Formierung und ihre strategischen Effekte als Phänomene für ihre Identifizierung heranzieht und man so das Fungieren rals< Dispositiv für eine Macht ausübende Instanz erschließen kann.« (a.a.O.: 9 [Herv. i. O.]) Eine sodann entstandene Multiperspektivität kann einerseits analytisch erfassen, »(a) wie und ob strategische Intentionen tatsächlich Wirkungen erzielen, (b) wie und ob Widerständigkeiten gegenüber Dispositiven mobilisiert werden sowie auch (c) wie und ob Dispositive von anderen (als ihren ursprünglich Formierenden) für andere (als die ursprünglich intendierten) Zwecke eingesetzt werden.« (Ebd.) Andererseits kann das Dispositiv als eine »Instanz< des Diskurses« (Keller 2007: 45 zit.n. Diaz-Bone \& Hartz 2017: 17 [Herv. i. O.]) die wechselseitigen Machtwirkungen »zwischen Diskursen und Praxisfeldern« (Diaz-Bone \& Hartz, 2017: 17) verdeutlichen und »die Infrastruktur der Diskursproduktion und Problembearbeitung« (ebd.) berücksichtigen. Das Dispositiv vermittelt also die »Verhältnisse, Relationen und Abhängigkeiten« (Truschkat 2017: 134), welche sich zwischen einem rationalisierten Diskurs und einer eingeleiteten Praxis abspielen (vgl. a.a.O.: 140). ${ }^{7}$

Schließlich, in Anlehnung an Gilles Deleuze, ließe sich metaphorisch "zwischen dem Dispositiv als >einer Art abstrakten Maschine « und den >konkreten Maschinen<, die jene verbindet « unterscheiden (Eugster 2013: 57 [Herv. i. O.]). Unter den konkreten Maschinen werden Mikro-Dispositive verstanden, d.h. "Kleinelemente wie z.B. Gebäude, Räume, Geräte und Praktiken.« (Ebd.) Doch überdies handelt es sich nicht nur um Teile einer größeren, "strategisch kohärenten sabstrakten Maschine« (a.a.O.: 58 [Herv. i. O.]), sondern darunter könnten auch »disparate Teile seiner diffusen, heterogenen Mannigfaltigkeit` (Deleuze 1996: 15) « verstanden werden, die sich »losgelöst von einem bestimmten Dispositiv-Zusammenhang« betrachten lassen (ebd. [Herv. i. O.]). So kann ein einzelnes Mikro-Dispositiv »sowohl mehreren Dispositiven angehören als auch seine Verbindung zu Dispositiven verlieren. Durch Umnutzungen können Mikro-Dispositive neu eingebunden werden und so gänzlich neuen Strategien dienen.« (Ebd.)

Mit einer solch erweiterten Perspektive werden die jeweiligen Teile eines Dispositivs nicht an ein Dispositiv festgebunden, sondern können mehreren Dispositiven anhören, diese bewirken, sich splittern, auf Zeit nicht aktiv sein, sedimentieren, wiederaufgenommen werden usw. »Eine solche Perspektive achtet zunächst 
darauf, welche Effekte die untersuchten Mikro-Dispositive bewirken, mit welchen diskursiven Codierungen sie in der zeitgenössischen Rezeption verknüpft werden und mit welchen anderen Mikro-Dispositiven und Diskursen sie allenfalls ein größeres dispositives Gefüge bilden.« (Ebd.) Sie werden indes analytisch nicht danach befragt, »welche Rolle sie in seinem positiven und Diskursen sie insgesamt eine Verkopplung eingehen.« (ebd. [Herv. i. O.])

\subsection{Dispositiv in Wissens- und Machtkonstellation}

Für die Diskursforschung spielt der Begriff des Dispositivs insofern eine bedeutende Rolle, als er die Diskursanalyse um weitere Elemente erweitern kann. Da beide Konzepte, der Diskurs und das Dispositiv »in hohem Maße übereinstimmen« (Jäger 2015: 76), kann das Dispositiv als ein prozessierender Zusammenhang von Wissen und gleichzeitig als eine Verfügungs-Macht analysiert werden.

\subsubsection{Dispositiv in Wissenskonstellation}

Als ein prozessierender Zusammenhang von Wissen ist das Dispositiv in drei Elementen des Sprechens/Denkens, des Handelns/Tuns und des materialisierten/vergegenständlichten Wissens zu analysieren: »Es geht also um einen Zusammenhang sprachlich performierter Diskurse (= Sprechen und Denken auf der Grundlage von >Wissen $\varsigma$ ), nicht-sprachlich performierter Diskurse (= Handeln auf der Grundlage von >Wissen`) und Sichtbarkeiten bzw. Vergegenständlichungen (als Resultate von Handeln auf der Grundlage von >Wissen`).« (a.a.O.: 113 [Herv. i. O.]) Im Fall der Dispositivanalyse müssen also drei Diskurs-Komponente - Sprach-Diskurse, Handlungs-Diskurse und Gegenständlichkeits-Diskurse - an ihr zugrundeliegendes (diskursives) Wissen rekonstruiert werden. Das Wissen kann in der Sprache zirkulieren ebenso wie in den Handlungen oder Gegenständen geäußert werden. Und gerade diese Vielgestaltigkeit des Wissens kann mit dem Konzept des Dispositivs besser erfasst werden (vgl. ebd.).

Zur Rekonstruktion dieser Äußerungen eignen sich »akribische Beobachtungen von Handlungen, die mit dem zu untersuchenden Gegenstand verbunden sind« (a.a.O.: 114) aber auch die Ermittlung des Wissens, das über sprachlichperformierte Diskurse zirkuliert, »also durch diverse Medien, Interviews mit den beteiligten Personen und mit Experten u. ä.« (a.a.O.: 115) Der nächste Schritt in der Dispositivanalyse besteht in dem Rekonstruieren und Feststellen der Beziehungen, die sich zwischen den jeweiligen Komponenten herausbilden: »In welcher Beziehung steht Handlungswissen, sprachlich performiertes Wissen und gegenständlich performiertes Wissen? [...] [W]ie kommunizieren diese drei Komponenten miteinander?« (ebd.) Analytisch gilt es deshalb, diese Beziehungen möglichst konkret zu rekonstruieren und ein Analyseraster zu entwickeln, 
"mit dem alle drei Komponenten daraufhin befragt werden können, inwiefern sie auch Verweise auf die sonstigen Komponenten enthalten. Solche Verweise werden gebündelt und den jeweiligen anderen Komponenten zugeordnet.« (Ebd.) Währenddessen müssen laut Jäger zwei methodologische Entwicklungen im Blick behalten werden. Zum einen ist das Dispositiv ein umkämpftes Feld, auf dem viele diskursive Kämpfer um die Vorherrschaft ringen. Andererseits ist es gleichzeitig eine Entwicklung per se, ein Konglomerat, das seine eigene innere Richtung und Strategie (auch des Widerstands) verfolgt. Illustrieren ließe sich ein solches Dispositiv am Beispiel des Krankenhauses. Dieses ist einerseits

»ein diskursives Kampffeld, andererseits ist es aber auch ein Widerlager mit einer eigenen, ihm inhärenten Strategie, sich gegen solche Entwicklungen zu wehren: Das Dispositiv lässt sich nicht einfach ummodeln; sein Sosein sperrt sich gegen Veränderungsversuche, die möglicherweise seine eigene Struktur und Funktion verändern oder sogar in Frage stellen. Wir haben es also einerseits mit Strategien der Kämpfenden zu tun, andererseits aber auch mit der Strategie des Krankenhaus-Dispositivs als solcher.« (a.a.O.: 116)

Beim Dispositiv handelt es sich also um eine unvorhersehbare (vgl. Rabinow 2004 zit.n. Mautz 2012: 162) und nicht vorher durchdachte Strategie, um eine Art von Konstellationen, die zwar »der Macht dienlich sein können, aber deren ১Organisation « nicht geplant war.« (Diaz-Bone 2006: 80 [Herv. i. O.]) Daher entstehen bei der Analyse mehrere Gefahren. Die eine Gefahr liegt darin,

»dass alles Mögliche als >Dispositiv erscheinen kann, solange man das Dispositivkonzept nicht in eine umfassendere theoretische Perspektive sowie in ein angemessenes Forschungsdesign einbettet, die zusammen erst klären, warum etwas überhaupt als Dispositiv (in welcher Konstellation, für was, für wen und wozu) fungiert bzw. fungieren kann. Eine weitere Gefahr besteht darin, einfach von vermeintlich gegebenen Dispositiven auszugehen, so als ob man sie einfach >vorfinden < könne und als ob sie dauerhaft beständig, für alle sichtbar und so auch vorab identifizierbar seien. Wenn man Dispositive von ihrer (strategischen) Wirkung her denkt, dann sind unbeständige Prozesse oder flüchtige Konstellationen genauso als Dispositive denkbar. Zudem ist es möglich, dass man überhaupt erst vom Effekt oder der Wirkung auf das Vorhandensein eines Dispositivs schließen kann, welches vorab nicht erkennbar war.« (Diaz-Bone \& Hartz 2017: 20 [Herv. i. O.])

Über dies hinaus muss für eine umfassende und vollständige Analyse der Kontext der Entwicklung des Dispositivs, d.h. seine (Vor-)Geschichte, gesellschaftstheore- 
tische Einbettung, Spezifika der Politik, des Landes, der Interessengruppen usw. berücksichtigt werden (vgl. Jäger 2015: 116). ${ }^{8}$

\subsubsection{Dispositiv in Machtkonstellation}

Außerdem lässt sich Dispositiv nicht nur als eine Wissens-, sondern auch als eine Machtformation analytisch untersuchen. So existiert das Dispositiv als eine »Verfügungs-Macht< mittels eines Fächers oder einer >Klaviatur quasi instrumenteller Optionen.«(Link 2007: 220 [Herv. i. O.]) Das damit einhergehende Problem, das weder Foucault selbst noch die darauffolgenden Untersuchungen gelöst bzw. gestellt haben, besteht laut Link in dem Verhältnis zwischen der disponierten und der disponierenden Subjektivität (vgl. ebd.). Die Macht oder die Verfügungs-Macht kann nur zwischen zwei Polen entstehen, demjenigen, der über sie verfügt, und demjenigen, über dem sie ausgeübt wird. Es handelt sich um eine Opposition »zwischen einem >objektiven instrumentellen Topik-Pol und einem >subjektiven Verfügungspol [...]. Mit Subjekt-Pol ist dabei die Subjektivität des >Disponierenden gemeint, d.h. des Verfügenden über das Dispositiv, des Strategen, des Mächtigen. Diese Subjektivität des Herren ist nun in den bisherigen Rekonstruktionen eigenartigerweise ausgespart zugunsten der alleinigen Subjektivität des Knechtes, konkret des Filmzuschauers im dunklen Saal, des Manipulierten, militärisch gesprochen des Soldaten. (ebd. [Herv. i. O.]) Wie Link weiter in Anlehnung an JeanLouis Baudry zeigt, sind jedoch beide Pole immerhin Teile des Ensembles, aus dem die Dispositive gebildet werden. Auch sie, diejenigen, die über die Strategie disponieren, »beherrschen keineswegs ihre Dispositive, auch sie sind genau genommen bloße Elemente, >Relays der Dispositive.« (a.a.O.: 22of [Herv. i. O.]) Die disponierten Subjektivitäten bilden hingegen »integrierende Elemente der instrumentellen Topik, über die die Klaviatur von Optionen der disponierenden Subjekte verfügen kann. Der Unterschied zwischen den disponierenden und den disponierten Subjekten, die beide im Dispositiv funktionieren, liegt genau darin, dass die letztgenannten keinen Zugang zur >Klaviatur haben.«(a.a.O.: 224 [Herv. i. O.]) Damit geht selbstverständlich die Frage einher, wie genau »Resistenz und Machtumsturz mittels der Dispositive« funktionieren (können) (a.a.O.: 234). Für Link geschehen der Machtumsturz und die mögliche Resistenz durch »das Spiel entgegengesetzter diskursiver Positionen« (a.a.O.: 236), das durch die Interdiskurse ${ }^{9}$ eröffnet wird.

8 Für weitere dispositivanalytische Schritte anhand einer erweiterten Diskursanalyse siehe ]äger 2015: $118 \mathrm{ff}$.

9

Die Interdiskurse werden nach Link als eine Kombination von Spezial- und Elementardiskursen generiert, und zwar so, »dass Verständlichkeit für eine allgemeingebildete Öffentlichkeit zustande kommt. (Link 2006: 19) Das, was in den Spezialdiskursen (naturwissenschaftlichen, human- und sozialwissenschaftlichen, kultur- und geisteswissenschaftlichen Diskursen und ihren Praktiken) ausgehandelt und am Wissen generiert wird, gliedert sich » abwärts vertikak in hierarchische Stufen (mit Anschluss an die svertikales, stratifikatorische Achse). 
Interdiskurse bilden diskurstheoretisch die Schnittstelle zwischen den (wissensgenerierenden) Spezialdiskursen und den (hegemonierten) Elementardiskursen. An dieser Schnittstelle werden die komplexen dispositiven Ensembles reduziert und subjektbezogen, sodass im Endeffekt die (Macht-)Verhältnisse kristallisiert und analytisch ersichtlich werden, wie z.B. die Verhältnisse zwischen Ärzten und Patienten, Polizisten und Kriminellen, Arbeitgebern und Arbeitnehmern usw. Erst dann leuchtet es im Hinblick auf die Möglichkeiten der Resistenz ein, »dass die Umwertung einer diskursiven Position auch die Umdrehung der Rollenverteilung zwischen disponierender und disponierter Subjektivität generieren kann: Dann werden im Extremfall Klienten zu Therapeuten, Arbeiter zu Ingenieuren, Laien zu Experten und Weiber zu Hyänen, d.h. zu revolutionären Politikerinnen.« (Ebd.) ${ }^{10}$

Die diskursanalytischen Anwendungen von Foucaults Dispositivbegriff liefern zahlreiche Hinweise für die Schärfung des Forschungsstils, mit dem die folgende Untersuchung an das Problem der Prävention psychischer Störungen, wie des Burnout-Syndroms, herangeht. Eine noch konkretere Präzisierung dieses Forschungsstils findet sich in seiner machtkritischen Rezeption wieder.

\subsection{Machtkritische Rezeption des Dispositivbegriffs}

Auch in der politikwissenschaftlichen Forschung wird die Dispositivanalyse dazu angewendet, »die Beziehungen zwischen Diskursen, Institutionen und vielfältigen Praktiken daraufhin zu untersuchen, ob und wie sich diese heterogenen Elemente zu einer kohärenten strategischen Formation verbinden. Damit ist gemeint, dass durch diese Elemente und die Beziehungen zwischen diesen Elementen Machtbeziehungen konstituiert oder reorganisiert werden, so dass die Effekte als kohärente, wenn auch subjektlose Strategie betrachtet werden können.« (Ziai 2005: 22) In Verbindung mit dem Konzept der Gouvernementalität und mit Foucaults drei Kernelementen der Analyse, ließe sich das Dispositiv im Trias Rationalität-Technologie-Subjektivierungsweise weiter präzisieren. So wird analytisch gefragt,

»erstens nach den Begründungen und Zielsetzungen, nach den Rechtfertigungsordnungen und Plausibilisierungsstrategien. Zweitens richtet sich das Augenmerk auf die Technologien des Regierens, auf jene Verfahren also, mit denen planvoll auf das Handeln von Individuen oder Cruppen eingewirkt wird

Oben befindet sich in dieser >vertikalen< Dimension eine mehr >elaborierte ( >informierteく, 'gebildete`, sintellektuelle`) Stufe und darunter eine mehr selementare` (`alltäglicheく, >populäre usw.).« (Link 2007: 232 [Herv. i. O.]) Die horizontale Achse bildet die Spezialdiskursedie Wissensteilung - ab, die vertikale Achse gibt demgegenüber die Elementardiskurse - die Machtverhältnisse - wieder (vgl. a.a.O.: 23off, insb. Abb. 3 und 4).

10 Diese Art der Umwertung liegt im Herz Foucaults Verständnis von mobilen, reversiblen und instabilen Machtbeziehungen (vgl. Foucault [1984] 2005b: 890). 
oder diese auf ihr eigenes Handeln einwirken. Drittens geht es schließlich um die Subjektivierungsweisen, die Art und Weise, wie die Programme des Regierens und Sich-selbst-Regierens die Individuen adressieren, um die ihnen eingeschriebenen Selbstbilder und Selbstformungspraktiken.« (Bröckling \& Peter 2017: 289)

Tatsächlich, indem sich die Macht in Foucaults Analysen immer auf konkrete Subjektkonstellationen bezieht, ist auch das letztendliche Ziel des Dispositivs »das Verhalten von Individuen so zu regulieren, dass die Verhaltensweisen in einem relationalen Verhältnis zum Regulierten stehen. Folglich produziert das Dispositiv Subjektivierungen und reagiert bzw. antwortet auf Subjektivierungen. "(Paulus 2015) ${ }^{11}$

Mit diesem »Blick auf Subjekte als >Fluchtlinien« von Dispositiven« (Diaz-Bone \& Hartz 2017:14 [Herv. i. O.]) kristallisiert sich das machtkritische Verständnis vom Dispositiv heraus, und zwar als Mittel dafür, die Produktion von Subjektivierungsweisen erfahrbar machen zu können. Als diskursive Akteure sind die Subjekte nämlich direkt daran beteiligt, wie die Dispositive funktionieren (können), was wiederum die Analyse der Machtbeziehungen klarer werden lässt. Forschungspraktisch wird dann zwischen den Subjektformierungen unterscheidet, die das Wissen darüber enthalten, »wer der einzelne im Verhältnis zu anderen sein soll, welche Praktiken dabei zu verfolgen sind und welche Bewertungen damit einherzugehen haben « (Bührmann \& Schneider 2012: 69), und Subjektivierungsweisen, die die über Dispositive produzierte und vermittelte Art und Weise darstellen, "wie sich Individuen im Verhältnis zu und im sozialen Austausch mit anderen bzw. mit der Welt selbst wahrnehmen, (leibhaftig) fühlen und in ihren verkörperten Praktiken mehr oder weniger habitualisiert präsentieren.«(a.a.O.: 60)

Die Dispositive funktionieren demnach als »sich institutionalisierende bzw. institutionelle Antworten auf bestimmte gesellschaftliche Problemlagen; sie reagieren auf und produzieren selbst gesellschaftlichen Wandel und zeitigen beabsichtigte, aber auch nicht beabsichtigte Wirkungen, Nebenfolgen innerhalb des gesamten Ensembles als Transformation von Machtbeziehungen und Herrschaftsstrukturen.«(a.a.O.: 93) Dafür müssen sie bestimmte Subjektmodi herstellen bzw. sich der bestehenden bedienen. Denn als Antworten auf gesellschaftliche Problemlagen organisieren sie die Machtverhältnisse und dienen in diesem Sinne als Regierungstechnologien. Demnach ließe sich auch das Dispositiv der Prävention als eine Regierungstechnologie begreifen und analytisch zugänglich machen. Die Operatio-

11 Stefan Paulus geht in Anlehnung an Foucault davon aus, dass das Dispositiv in der Zeit »zum Wahrnehmungs-, Denk-, Entscheidungs- und Handlungsschemata für Subjekte [wird]. Es bringt Subjekte hervor bzw. reguliert diese, indem die Einrichtungen durch die jeweiligen Regelsysteme stabilisierenden Einfluss auf das Verhalten von Individuen und Cemeinschaften haben (vgl. Foucault 1978).«(Paulus 2015) 
nalisierung der Analyse des Präventionsdispositivs wird im nächsten Teil verdeutlicht.

\section{Operationalisierung der Dispositivanalyse}

Werden nun die bisherigen Ausführungen zum Hintergrund des DispositivBegriffs bei Michel Foucault sowie zu seiner späteren wissenschaftlichen Rezeption zusammengeführt, so ergeben sich daraus folgende Hinweise für die Untersuchung des Präventionsdispositivs.

\subsection{Analyseschritte}

Die Analyse des Präventionsdispositivs besteht aus folgenden Schritten:

- Erstens besteht das Dispositiv der Prävention aus mehreren Elementen, und zwar - hier nach Bührmann und Schneider (2012) - den Diskursen, den nichtdiskursiven Praktiken, den Vergegenständlichungen und den Subjektivationen (s. Abb. 1).

- Zweitens bestehen zwischen diesen Elementen wechselseitige Beziehungen, d.h. dass die Elemente aufeinander verweisen und ein mehr oder weniger homogenes Ensemble bilden. Diese müssen entsprechend rekonstruiert bzw. während der Analysen verdeutlicht werden.

- Drittens, dieses dispositive Ensemble existiert nicht bloß in Raum und Zeit, sondern ist in komplexe Zusammenhänge und soziale Wandlungen eingebunden und hat auch dementsprechend unterschiedliche Wirkungen und (Neben-)Effekte. Die gesellschaftstheoretische Kontextualisierung des Präventionsdispositivs muss es daher in die gegenwärtigen Entwicklungen und gesellschaftlichen Wandlungen einbetten, damit es intelligibel und zugänglich wird.

- Viertens und in Bezug auf das Zentrum der Dispositivanalyse - der Diskursanalyse - stellt das Dispositiv der Prävention eine Erweiterung des Präventions-Diskurses in dem Sinne dar, als es nicht nur »das NichtDiskursive, das Gegenständliche, das (praktische) Tun als solches« pointiert (Bührmann \& Schneider 2012: 93), sondern zu der Auseinandersetzung mit der Frage zwingt, »was aus diskursiv vermittelten Wissensordnungen inwieweit >wirk-liche ( (und insofern >machtvolle`) Effekte zeitigt, als dass es in seiner kollektiven wie individuellen Vermittlung im Selbst- wie Weltbezug handlungswirksam wird und dadurch (erst) auf jene Wissensordnungen rückwirken kann.« (ebd. [Herv. i. O.]) Eine solch erweiterte Analyse richtet sich dann auf die Ermittlung von Tatsachen, die diskursiv erzeugt wurden und 